\title{
ANALYSE DES PRATIQUES \\ DE RSE DES CLUBS SPORTIFS PROFESSIONNELS FRANÇAIS
}

\begin{abstract}
Aurélien FRANÇOIS
Docteur - Université de Bourgogne - Laboratoire Socio-Psychologie et Management du Sport (SPMS)

Adresse professionnelle : Université de Bourgogne, Faculté des Sciences du Sport, Laboratoire Socio-Psychologie et Management du Sport (SPMS) - EA 4180, Campus Universitaire, BP 27877, 21078 Dijon Cedex aurelien.francois@u-bourgogne.fr
\end{abstract}

\section{Emmanuel BAYLE}

Professeur - Institut des Sciences du Sport de I'Université de Lausanne (ISSUL)

Adresse professionnelle : Quartier UNIL-Mouline, Bâtiment Géopolis, Bureau : 5442, CH-1015 Lausanne emmanuel.bayle@unil.ch

\section{RÉSUMÉ}

Autrefois réservé à la grande entreprise, le concept de Responsabilité Sociétale des Entreprises (RSE) s'étend aujourd'hui à l'ensemble des organisations. Dans cette optique, cet article s'intéresse aux déterminants et aux modalités de mise en œuvre des pratiques de RSE dans les clubs sportifs professionnels français. À partir d'un cadre d'analyse permettant d'associer les approches institutionnelles et stratégiques en matière de RSE, nous montrons la difficulté pour ces organisations " hybrides » de trouver un équilibre entre les logiques d'action qui les animent. Les résultats montrent que les quatre cas de clubs étudiés (de basket-ball et de football) exercent des pratiques de RSE revêtant surtout une forme cosmétique. L'émergence de nouvelles formes de RSE laisse pourtant entrevoir des pratiques plus intégrées à même de réduire la tension entre les logiques économiques et sociales de ces organisations.

Mots clés : Responsabilité Sociétale de l'Entreprise (RSE), clubs sportifs professionnels, stratégie, légitimité.

\section{ABSTRACT}

In the past reserved to firms, the concept of Corporate Social Responsibility (CSR) is spreading today to all organizations. This paper thus tackles the determinants and the implementation procedures of CSR within French professional sport clubs. Using a framework of analysis, which combines institutional and strategic approaches, we highlight the difficulty for those "hybrid" organizations to find a balance between the logics they face. The results show that the four clubs we studied (basketball and football) develop CSR practices mainly for cosmetic purposes. However the rise of new CSR forms let us glimpse a more integrated practice which could ease the tension between economic imperatives and social expectations.

Keywords: Corporate Social Responsibility (CSR), professional sport clubs, strategy, legitimacy. 


\section{INTRODUCTION}

"Les clubs de foot ne doivent pas se méprendre sur ce quils sont. Ils ressemblent moins à une grande entreprise qu'à un musée : une organisation à l'esprit civique qui a pour but de servir la communauté tout en restant raisonnablement solvable. Cet objectif peut paraître modeste mais très peu de clubs parviennent à l'atteindre" (Kuper \& Szymanski, 2012: 75).

Cette citation, généralisable à l'ensemble des clubs sportifs professionnels, commande à ces organisations un retour à plus de citoyenneté. Devant les nombreuses dérives éthiques (argent roi, corruption, dopage...) relayées massivement par les médias, la légitimité des clubs professionnels est, en effet, régulièrement remise en cause. Dans ce contexte, la question de la Responsabilité Sociétale des Entreprises (RSE) ou des Organisations (RSO) s'est invitée à l'agenda de la recherche en management du sport dès la fin des années 2000 (Bradish \& Cronin, 2009; Godfrey, 2009). En 2009 et 2010 paraissaient ainsi successivement deux numéros spéciaux dans Journal of Sport Management et dans Journal of Management and Organization compilant une quinzaine d'articles sur le thème de la RSE dans le sport. Ligues et clubs professionnels nord-américains ont depuis été l'objet d'une littérature importante en la matière (Babiak \& Wolfe, 2009 ; Paramio-Salcines, Babiak \& Walters, 2013 ; Sheth \& Babiak, 2010 ; Walker, Kent, \& Vincent, 2010). En France, les rares productions scientifiques sur le thème de la RSE dans le sport ont mis en évidence le stade embryonnaire de son développement (Bayle et al., 2011). L'étude de la RSE des clubs professionnels s'impose ainsi au regard de la transposition novatrice du concept au mouvement sportif. Elle participe également à l'extension de son champ d'application. Autrefois cantonnée aux grandes sociétés cotées, l'étude de la RSE se porte ainsi sur les organisations en général à travers le concept de RSO (Bento, 2009 ; Paradas, 2008). Les clubs sportifs professionnels dont les tailles ${ }^{1}$ relèvent de celles de petites et moyennes entreprises apparaissent ainsi comme un nouveau terrain fécond de recherche. En outre, nous situons notre contribution dans la lignée des travaux sur les organisations " hybrides " (Battilana \& Dorado, 2010). L'hybridité est appréhendée comme étant à la base du management des organisations du domaine sportif dont les clubs sportifs professionnels constituent le cour (Bayle, 2007). En effet, les logiques d'action de ces organisations relèvent de différents « registres" (associatif, marchand et public) comme le montre leur financement privé (droits TV, sponsoring) et public (subventions, mise à disposition de stade...), la mixité de leur personnel (salariés et bénévoles) et les finalités auxquelles elles répondent (profit versus objectifs extra-économiques de type sociétaux).

L'objectif de cet article est d'analyser le degré d'engagement des clubs professionnels français dans la voie de la RSE afin de révéler les différentes formes de pratiques de RSE existantes. Nous exposons en premier lieu le cadre théorique mobilisé combinant approches néo-institutionnelle (Campbell, 2007) et stratégique (Porter \& Kramer, 2006) dont l'association rend compte de la tension entre les différentes logiques d'action parcourant les organisations sportives professionnelles. Nous proposons ainsi un cadre d'analyse des pratiques de RSE composé de sept dimensions (facteurs d'influence, objectifs, parties prenantes, ressources, outils de gestion, actions et évaluation). À partir de ce cadre, nous étudions les pratiques de RSE de quatre cas de clubs sportifs professionnels français (Villeurbanne et Chalon sur Saône en basket-ball et Auxerre et Lyon en football). Puis, nous traitons des résultats en confrontant les observations empiriques au cadre d'analyse, en mettant en évidence le caractère cosmétique de la RSE symbolisée par une pratique essentiellement axée sur la communication externe des actions sociétales. Enfin, nous discutons, à la lumière d'un projet de taxonomie (Martinet et Payaud, 2008), du niveau d'engagement des organisations dans la voie de la RSE. A ce titre, nous envisageons les conditions du passage d'une RSE cosmétique à une RSE intégrée, cette dernière permettant de concilier les différentes logiques d'action qui animent ces organisations et ainsi de réduire le découplage entre ces logiques (Crilly, Zollo \& Hansen, 2012). C'est dans cette optique que la RSE peut être envisagée comme une nouvelle composante stratégique à même de dépasser les résultats sportifs et économiques dans l'évaluation de la performance des clubs sportifs professionnels.

\section{ANCRAGES THÉORIQUES DE L'ÉTUDE}

En synthétisant les différents courants théoriques organisationnels, Desreumaux (2005) dresse une double interprétation du comportement des organisations. D'une part, la vision structurelle explique que les décisions au sein d'une organisation sont le résultat de forces impersonnelles et exogènes. D'autre part, la vision stratégique attribue aux acteurs détenant le pouvoir dans les organisations la prise de

\footnotetext{
1. La taille des clubs sportifs professionnels peut aller de quelques salariés lorsque seuls les joueurs et quelques personnes du staff administratif ont un contrat de travail jusqu'à une centaine de salariés lorsque l'organisation est beaucoup plus développée (exemple de l'Olympique Lyonnais dans les cas présentés par la suite)
} 
décision des orientations stratégiques sur la base de choix rationnels et délibérés. La première vision est envisagée sous le prisme de la théorie néoinstitutionnelle sociologique qui conçoit les organisations comme encastrées dans la société et voit en la RSE un moyen d'augmenter leurs légitimités au sein de leurs environnements. La seconde conception s'inscrit dans une approche proactive visant à développer, à travers une RSE "stratégique ", le potentiel de création de valeur de l'organisation (Porter \& Kramer, 2006, 2011 ; Vogel, 2005). Depuis plusieurs années, les auteurs en sciences de gestion s'accordent à dire qu'il convient de mettre en interaction les caractères déterministe et nondéterministe de ces deux visions afin de mieux comprendre le comportement des organisations (Judge \& Zeithaml, 1992).

\subsection{L'approche néo-institutionnelle de la RSE}

La théorie néo-institutionnelle (TNI) pose l'hypothèse qu'une organisation est encastrée non pas uniquement dans son environnement technique mais aussi institutionnel (Scott, 1995). La recherche de légitimité permet d'augmenter les ressources des organisations, afin que celles-ci puissent parvenir à survivre dans leurs environnements concurrentiels (Meyer \& Rowan, 1977 ; Suchman, 1995). Si leurs capacités à contrôler leurs activités formelles constituent un premier pas vers le succès, les organisations ont donc besoin de se conformer aux mythes institutionnels afin que leurs activités soient jugées conformes par les acteurs de leurs environnements (Meyer \& Rowan, 1977 ; DiMaggio \& Powell, 1983). L'ouvrage de Bowen (1953), cité comme le point de départ de la conception moderne de la RSE (Carroll, 1979, 1999), consacre une vision institutionnaliste du concept. Dès lors, les nombreuses contributions qui ont par la suite appréhendé la RSE sous cet angle constituent plus un retour aux sources qu'un nouveau prisme de lecture conceptuelle (Acquier \& Gond, 2007). Ainsi, Campbell (2007) met en avant la présence de nombreuses variables institutionnelles modérant l'idée selon laquelle la stratégie de RSE est mise en place à des fins uniquement économiques. Parmi elles figurent l'intensité de la régulation publique/privée autour de la RSE, la présence d'ONG ou de normes sociales institutionnalisées ainsi que des dialogues organisés autour de la thématique de la RSE avec les principales parties prenantes de l'organisation. Dans la même veine, les travaux de Matten et Moon (2008) ont souligné l'importance des variables institutionnelles afin de montrer que la RSE aux Etats-Unis est exercée de façon explicite et stratégique alors qu'en Europe elle l'est de façon beaucoup plus implicite. Enfin, des travaux francophones se sont emparés de la vision néo-institutionnelle pour expliquer l'adoption et la diffusion des pratiques de RSE dans le domaine des chartes éthiques (Le Borgne-Larivière, Mauléon, \& Schier, 2009), des normes environnementales (Boiral, 2006) ou de l'engagement sociétal des multinationales (BorchaniMaktouf, 2010).

\subsection{L'approche stratégique de la RSE}

Alors que l'approche néo-institutionnelle se distingue par un niveau de déterminisme élevé (Hasselbladh \& Kallinikos, 2000), la vision stratégique s'y oppose. Celle-ci suppose que les acteurs détenant le pouvoir au sein des organisations ont toute latitude pour prendre des décisions stratégiques. Cette idée est notamment supportée dans la théorie du choix stratégique (Child, 1997) mettant en avant le pouvoir d'influence dont disposent les managers pour orienter leurs organisations. Cette approche a connu un regain d'intérêt dans le champ de la RSE (Lee, 2008) avec la publication d'articles montrant que l'intégration de pratiques de RSE peut développer le potentiel de création de valeur des organisations (Husted et Allen, 1998 ; Porter \& Kramer, 2006). Enfin, de récents apports théoriques (Porter et Kramer, 2006, 2011) sont riches d'enseignements sur la façon dont une entreprise peut contribuer à son développement tout en participant à l'amélioration des conditions de vie dans son environnement. C'est sur la base de ces travaux qu'ont été réalisées de nombreuses études en matière de stratégies de RSE, notamment dans le cas des grandes entreprises (Martinet \& Payaud, 2008 ; McWilliams, Siegel \& Wright, 2006, 2011).

Du point de vue théorique, les courants stratégique et institutionnel se situent dans des niveaux d'analyse distincts, le premier s'intéressant plus aux conséquences et le second aux causes de la RSE (Aguinis \& Glavas, 2012). Néanmoins, de nombreux auteurs ont pris le pari de ne pas cliver ces deux registres d'actions en matière d'engagement social (Borchani-Maktouf, 2010 ; Dupuis, 2011 ; Suchman, 1995). C'est de la complémentarité entre ces deux courants que tire l'origine des dimensions du cadre d'analyse présenté ci-dessous.

\section{CADRE D'ANALYSE}

Le cadre d'analyse (figure 1) met en évidence les causes d'engagement des clubs sportifs professionnels dans la RSE et sa mise en ouvre. La première étape introduit l'identification de facteurs d'influence comme une phase essentielle de l'analyse. Elle interroge ainsi les raisons pour lesquelles les organisations se comportent de manière responsable (Capron et Quairel-Lanoizelée, 2010). La réponse à cette question introduit un éventail de théories sous- 
jacentes au construit du concept de RSE. Parmi elles, l'approche néo-institutionnelle évoque la recherche de légitimité comme un leitmotiv fort de l'adoption et la réalisation de pratiques de RSE. L'approche contractuelle à l'origine de la "triple bottom line » et de la notion de performance sociétale (Wood, 1991) et celle des parties prenantes (Freeman, 1984) repose, quant à elle, sur l'idée d'un lien positif entre RSE et performance organisationnelle et se situe dans une perspective stratégique. La seconde étape traite de la mise en ouvre de la RSE dans l'organisation des clubs. Sa construction est directement inspirée de la méthode PDCA (Plan-Do-Check-Act) utilisée dans le déploiement de la norme ISO 26000 (QuairelLanoizelée, 2011). Cette méthode a été consacrée dans de nombreuses contributions dans le champ de la RSE. Celle de Maon, Lindgreen et Swaen (2009) propose un cadre d'analyse intégrateur de la RSE à partir d'une approche mixant cadres théoriques et observations empiriques dont nous nous sommes inspirés pour établir notre cadre d'analyse.

\subsection{L'identification des facteurs d'influence des pratiques de $R S E$}

Depuis son émergence en tant que concept académique (Bowen, 1953), la RSE est définie par son caractère volontaire ce qui s'est traduit par une multiplication de normes et de labels attestant, de façon non-contraignante, la bonne conduite des organisations. En France, à l'exception de l'article 116 de la loi NRE de 2001 puis l'article 225 du Grenelle 2 de l'Environnement de 2012, dont les périmètres d'application restreints n'imposent qu'un reporting sociétal, mais pas de nouvelles actions de fond en matière de RSE, aucune législation ne contraint les organisations en la matière. L'intérêt d'étudier les déterminants de l'engagement des organisations dans la RSE constitue dès lors une thématique largement abordée. Certains travaux ont utilisé le terme de " facteurs d'influence » (Pastore-Chaverot, 2011) dont nous reprenons la terminologie pour désigner les causes de la réalisation de pratiques de RSE. Dans le contexte du sport professionnel, certains auteurs se sont emparés tour à tour des approches néoinstitutionnelle et stratégique afin d'étudier ces facteurs d'influence (Babiak \& Wolfe, 2009 ; Babiak \& Trendafilova, 2011). S'appuyant sur le cadre d'analyse d'Oliver (1991) reposant sur les hypothèses convergentes de la théorie néo-institutionnelle et de la théorie de la dépendance envers les ressources (Pfeffer \& Salancik, 1978), Babiak et Wolfe (2009) montrent ainsi que les déterminants des pratiques de RSE dans le sport professionnel peuvent être expliqués par ces deux courants. À partir d'entretiens menés auprès de dirigeants des ligues professionnelles nordaméricaines, ces auteurs mettent ainsi en avant la prégnance de déterminants externes (pressions des parties prenantes externes) et internes (ressources disponibles) expliquant l'adoption de pratiques de RSE dans les clubs.

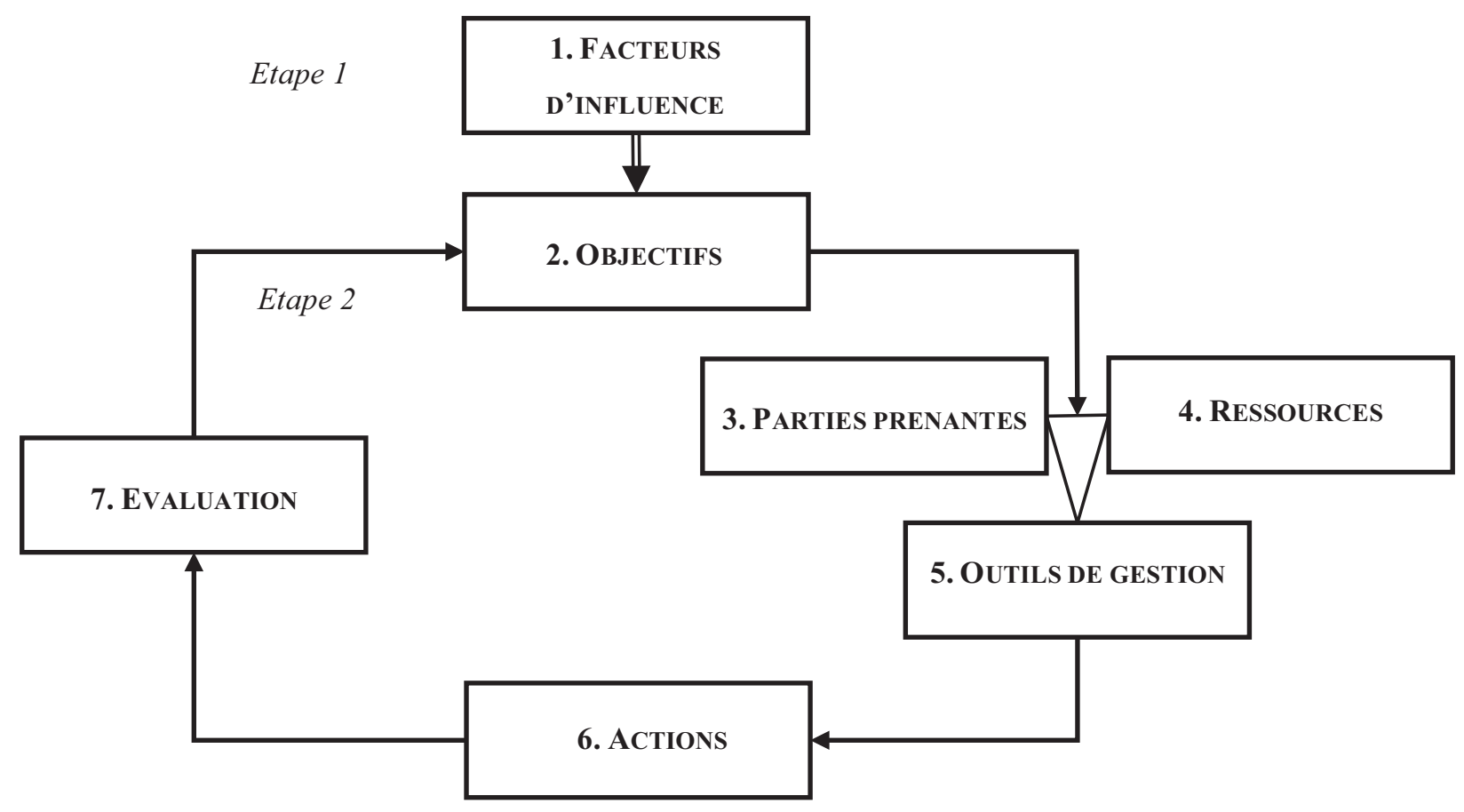

$\Longrightarrow$ Relation inter-étapes

Liens inter-dimensionnels de l'étape de mise en œuvre de la RSE

Figure 1 : Cadre d'analyse des pratiques de RSE des clubs sportifs professionnels. 


\subsection{L'implémentation des pratiques de RSE}

À l'instar des travaux de Maon, Lindgreen et Swaen (2009), chaque phase de la méthode PDCA a été traduite en termes managériaux (objectifs-moyensactions-évaluation), puis introduite comme dimension du cadre d'analyse. En regardant de plus près l'étape de la mobilisation des moyens, on peut distinguer les parties prenantes associées au processus de mise en œuvre de pratiques de RSE, les ressources organisationnelles mobilisées pour la réalisation de ces pratiques et les outils de gestion permettant de combiner l'ensemble des parties prenantes et les ressources associées. La théorie des parties prenantes (Freeman, 1984) qui constitue le corpus dominant du champ de la RSE apparaît ici incontournable. L'une des spécificités des clubs sportifs professionnels provient justement de la nécessité d'interagir au sein d'un réseau important de parties prenantes (Babiak \& Wolfe, 2009). La plupart des contributions articulant sport et RSE préconisent, d'ailleurs, d'intégrer ces multiples parties prenantes dans le management des clubs sportifs professionnels (Breitbarth \& Harris, 2008 ; Walters \& Chadwick, 2009). Certains vont jusqu'à considérer que les critères spécifiques de l'activité sportive (passion sportive, modèle économique reposant sur un financement public important...) nécessitent un management spécifique des parties prenantes dont les intérêt sont plus en lien avec la RSE (Babiak \& Wolfe, 2009 ; Smith \& Westerbeek, 2007). La dimension des ressources est issue du courant " resource-based view " dont la transposition au concept de RSE a été prégnante ces dernières années (Branco \& Rodrigues, 2006 ; McWilliams \& Siegel, 2011). Celle-ci est liée à la façon dont les parties prenantes s'approprient les ressources produites par l'organisation, en fonction du pouvoir qu'elles exercent sur cette dernière. Selon cette approche, le développement des pratiques de RSE dépend surtout des ressources dont les organisations disposent et de la manière dont elles les mobilisent. Maltese (2009) montre ainsi que les organisations sportives professionnelles se construisent autour de ressources et de compétences clés que sont la réputation, les réseaux relationnels, les supports physiques et les partenariats. La dernière dimension, celle des outils de gestion, se situe au croisement des théories instrumentales et des pratiques managériales. La littérature sur les outils de gestion de la RSE a été peu intégrée à celle de la RSE à l'exception de quelques travaux (Acquier, 2007). Les nombreuses interactions entre parties prenantes et ressources affectées en matière de RSE ont incité certains dirigeants de clubs sportifs professionnels à instrumentaliser et rationnaliser leurs pratiques autour de documents internes (réalisation d'énoncés de valeurs, de chartes éthiques...) ou de structures permettant de piloter la politique de mécénat des clubs.

\subsection{Un cadre d'analyse de I'hybridation des logiques d'actions en matière de RSE}

L'articulation entre les étapes d'identification des déterminants de la RSE et de leur mise en ouvre établit un lien entre causes et conséquences de la réalisation de pratiques de RSE (Aguinis \& Glavas, 2012). Elle constitue un premier niveau d'analyse de l'hybridation des logiques d'actions des clubs en matière de RSE. Le degré d'alignement entre ces deux étapes permet de mesurer les formes de couplage/découplage entre une RSE "déclarée » et une RSE "pratiquée "(Dupuis, 2011 ; Gond \& Igalens, 2012). Le lien entre les facteurs d'influence et les objectifs permet ainsi d'interroger les logiques d'action mises en ouvre dans les organisations à la lueur des cadres théoriques institutionnels et stratégiques. La RSE peut soit s'entendre comme une pratique en cours d'institutionnalisation dont la mise en œuvre relève de forces institutionnelles exogènes aux clubs, soit être le fruit d'une vision stratégique suite à la prise en compte des intérêts des parties prenantes influentes, des ressources disponibles à sa réalisation (Babiak \& Wolfe, 2009) ou de l'arrivée d'outils de gestion accélérant le processus d'adoption des pratiques de RSE (Acquier, 2007). Le cadre d'analyse constitue ainsi un moyen de confronter facteurs d'influence identifiés et pratiques observées et d'analyser les dynamiques de couplage ou de découplage à l'œuvre dans l'organisation.

Le second niveau d'analyse de l'hybridation des logiques d'action est mesuré à travers la cohérence inter-dimensionnelle du processus d'implémentation de la RSE (objectifs-moyens-actions et évaluation). Dans la tentative de mesurer le degré d'engagement des clubs en matière de RSE, l'analyse permet de confronter les niveaux de performance de la RSE dans chacune des dimensions de la seconde étape. Est mesuré ici l'alignement (ou non) entre objectifs, moyens mis en ouvre, action et évaluation afin de faire apparaître à nouveaux des logiques de couplage (ou découplage) d'une RSE " déclarée » et "pratiquée ».

\section{ETUDES DE CAS ET MÉTHODE D'ANALYSE ASSOCIÉE}

Notre posture méthodologique se situe dans une approche abductive de l'objet de recherche étudié. Nous avons opté pour la méthode des études de cas multiples (Yin, 2003) dont l'une des finalités est de pouvoir confirmer un début de théorisation (Glaser \& Strauss, 1967). Les études de cas menées nous ont ainsi permis de confronter les observations empiriques collectées aux dimensions du cadre d'analyse construit à partir d'une revue de la littérature en matière de RSE. 
Nos cas se sont centrés sur deux clubs de basket-ball (Chalon sur Saône et Villeurbanne) et deux clubs de football (Auxerre et Lyon). Le choix de cet échantillon s'est opéré sur la base des travaux d'Eisenhardt (1989) recommandant d'étudier a minima 4 cas pour pouvoir tirer des conclusions généralisables et ainsi augmenter la validité externe de l'étude. Ce nombre seuil nous a également été dicté par des considérations pragmatiques et notamment par la proximité géographique avec les lieux d'études (régions Bourgogne et Rhône-Alpes). Malgré cette limite, la diversité des modèles organisationnels sur lesquels a été constitué chacun des cas illustre le caractère hybride ainsi que la diversité juridique et économique des clubs sportifs professionnels français. L'analyse des pratiques de RSE s'est portée sur les structures commerciales (sociétés sportives gérant l'équipe professionnelle), associatives (association support obligatoire gérant la partie amateur du club) ainsi que celles dédiées au domaine social (fondations, fonds de dotation, associations...).

\subsection{Présentation des cas}

L'Olympique Lyonnais (OL) est un club de football constitué depuis 1999 en une holding (OL Groupe) possédant des parts de capital dans neuf entreprises filiales. En 2010, OL Groupe employait 248 salariés et générait un chiffre d'affaires de 160 millions d'euros. Depuis 2007, ce groupe a investi les marchés financiers afin de lever des fonds pour son nouveau stade dont l'inauguration est prévue en 2015. La cotation a fait entrer ce club dans le champ de la loi NRE, modifiée ensuite par la loi Grenelle II, et l'a obligé à intégrer des informations sociales et environnementales dans son rapport d'activité. En 2007, avec l'apport de sponsors, l'OL a créé une fondation d'entreprise (OL Fondation) afin de formaliser sa politique de mécénat menée depuis les années 2000 ainsi qu'un fonds de dotation en 2009. L'Association de la Jeunesse Auxerroise (AJA) est un club de football atypique pour deux raisons. D'abord, sa société commerciale, créée en 2000 et gérant la partie professionnelle, est contrôlée à 99\% par l'association support du club. De même, l'AJA est un des rares clubs en France à être propriétaire de son stade. La section football générait pour la saison 2009-2010 des produits d'activités à hauteur de 41 millions d'euros et employait environ 50 salariés. En matière d'initiatives sociales, l'AJA s'est joint à l'association Famille AJA fondée à partir du programme du même nom dans les années 2000 par le président de l'un des clubs de supporters. Cette initiative promeut les valeurs citoyennes auprès des jeunes supporters via notamment la mise en place de conférences citoyennes.
L’Association sportive de Villeurbanne éveil lyonnais (Asvel) est un club de basket-ball faisant partie d'une holding. Il est la propriété de trois actionnaires principaux dont Tony Parker, célèbre basketteur de NBA, arrivé en 2009 dans le capital du club. L'Asvel générait des produits d'activités s'élevant à 6,7 millions d'euros et employait une trentaine de salariés pour la saison sportive 2009-2010. Historiquement très liée, à la ville de Villeurbanne, l'Asvel réalise des actions sociales visibles depuis le début des années 2000. En 2007, ce club a créé une association citoyenne (Esprit Sport) qui met en ouvre divers programmes communautaires autour de la santé, du lien social et de la responsabilité sociétale. L'Elan Chalon est un club de basket-ball fondé en 1970. Du point de vue juridique, l'Elan Chalon est une société d'économie mixte. Sous ce statut, les collectivités territoriales et l'association support du club sont obligées de détenir conjointement plus de la moitié du capital du club. L'Elan Chalon générait des produits d'activité d'environ 3,7 millions d'euros et employait une trentaine de salariés pour la saison sportive 2008-2009. Ses actions sociales et citoyennes sont regroupées dans le programme "Elan pour Tous " dont l'objectif est d'exploiter l'image des joueurs à des fins citoyennes.

\subsection{Collecte des données}

Nous avons procédé à une triangulation des sources (Eisenhardt, 1989 ; Yin, 2003), afin d'augmenter la validité de notre recherche. Miles et Huberman (2003) préconisent de collecter différents types d'informations à partir de différentes sources ce que nous avons fait, en collectant un ensemble de données primaires et secondaires depuis le début des années 2000, au moment où se sont formalisées les premières pratiques de RSE des clubs enquêtés. Les données primaires ont été recueillies à travers la passation d'entretiens semi-directifs menés entre 2009 et 2011 auprès d'acteurs internes des clubs ayant une vision globale de leurs pratiques de RSE (annexe 1). La première question du guide d'entretien, interrogeant les déterminants de l'adoption de pratiques de RSE (étape 1), a permis d'alimenter la première dimension de notre cadre d'analyse. Puis était abordée la question de la mise en ouvre des pratiques de RSE (étape 2) autour des quatre thèmes consacrés dans la méthode PDCA (objectifs, mise en œuvre, actions et évaluation) portant sur les six dimensions restantes. Nous avons ensuite interrogé les principales parties prenantes associées à ces pratiques en distinguant celles intervenant dans le processus de mise en ouvre (acteurs internes des clubs, sponsors, pouvoirs publics) de celles qui en bénéficient (associations et organismes parrainés) (tableau 1). L'analyse des données issues de sources secondaires internes 
(rapports financiers et sociaux, actualités des sites internet...) et externes (convention de partenariats privées et publiques...) nous a permis de confirmer, d'atténuer ou de réfuter les propos des entretiens.

L’ensemble des entretiens a été retranscris puis analysé dans la lignée des travaux sur l'analyse de contenu conceptuelle consistant à décrypter le contenu des messages à partir de procédures objectives établies (Bardin, 2007). Les données ont été analysées manuellement en extrayant le sens des principales propositions utilisées par les acteurs interrogés et ce, de façon itérative, en opérant un processus d'aller et retour entre cadre théorique et observations empiriques. Pour chacune des dimensions du cadre d'analyse renseignées, la classification des réponses s'est opérée en distinguant les différents degrés d'intensité de l'engagement des clubs dans la voie de la RSE. Nous avons retenu tour à tour la primauté des facteurs internes face aux facteurs externes dans l'étape d'identification des déterminants de la RSE et le caractère stratégique symbolisé entre autre par le degré d'alignement ${ }^{3}$ entre les dimensions restantes (objectifs, parties prenantes, ressources, outils de gestion, actions et évaluation...).

\section{RÉSULTATS : LA LÉGITIMITÉ COMME LEITMOTIV PRINCIPAL DES PRATIQUES DE RSE MENÉES}

Les données obtenues pour chaque dimension du cadre d'analyse (tableau 2) introduisent deux résultats principaux. Le premier est la pression intense des partenaires publics à l'origine des pratiques de RSE lesquelles présentent un fort degré d'isomorphisme entre elles notamment autour d'une dimension sociale largement développée. Le second, fortement lié au premier, est la recherche de légitimité auxquelles répondent les finalités des pratiques mises en œuvre. Ces dernières se caractérisent par une pratique légère et symbolique de la RSE sans que celle-ci ne soit réellement envisagée comme composante stratégique de l'organisation.

\begin{tabular}{|c|c|c|}
\hline Cluubs & $\begin{array}{l}\text { Nombre D'ACTEURS } \\
\text { INTERROGÉS }\end{array}$ & Rôtes' \\
\hline ELAN CHALON & 11 & $\begin{array}{l}\text { - General Manager } \\
\text { - Acteurs internes (Président de l'association, entraîneur) (2) } \\
\text { - Partenaires privés (Sponsors privés) (4) } \\
\text { - Pouvoirs publics (3) } \\
\text { - Organisation soutenue (Institutrice d'une école publique) (1) }\end{array}$ \\
\hline ASVEL & 5 & $\begin{array}{l}\text { - Directeur Administratif } \\
\text { - Acteur interne (Chargée de projet de l'association Esprit Sport) (1) } \\
\text { - Pouvoirs publics (2) } \\
\text { - Association parrainée (1) }\end{array}$ \\
\hline AJA & 6 & $\begin{array}{l}\text { - Directeur Marketing et Communication } \\
\text { - Acteur interne (Président de l'association) (1) } \\
\text { - Partenaires privés (Chargé de projet à la Fondation du Football et } \\
\text { Président de l'association Famille AJA) (2) } \\
\text { - Pouvoirs publics (2) }\end{array}$ \\
\hline $\mathrm{OL}$ & 9 & $\begin{array}{l}\text { - Secrétaire Général d'OL Fondation } \\
\text { - Partenaires privés (Chargé de projet à la Fondation du Football et } \\
\text { sponsors privés engagés dans la fondation) (3) } \\
\text { - Pouvoirs publics (2) } \\
\text { - Associations parrainées (3) }\end{array}$ \\
\hline
\end{tabular}

Tableau 1 : Parties prenantes interrogées des quatre clubs étudiés et rôles associés.

2. Les rôles mis en gras correspondent aux entretiens centraux menés auprès des acteurs ayant une vision globale des pratiques de RSE et les chiffres entre parenthèses au nombre d'entretiens réalisés avec les autres acteurs.

3. Autrement dit, un haut niveau d'objectifs déclarés en matière de RSE doit théoriquement aboutir à une mobilisation importante de parties prenantes, de ressources et d'outils de gestion conduisant à la réalisation d'actions de RSE sociétales dont l'évaluation peut être source d'un retour sur les objectifs initiaux. 


\begin{tabular}{|c|c|c|c|c|c|c|c|}
\hline $\overrightarrow{0}$ & 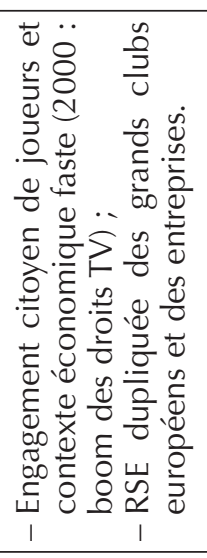 & 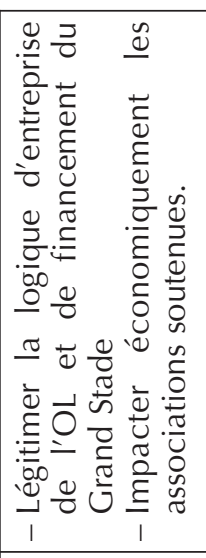 & 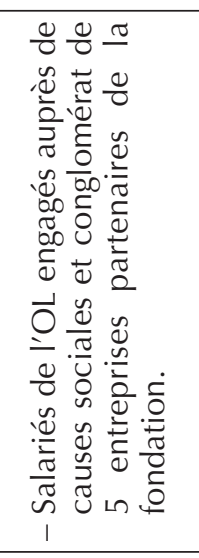 & 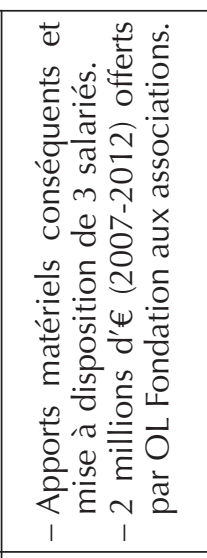 & 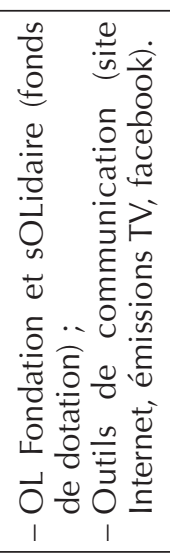 & 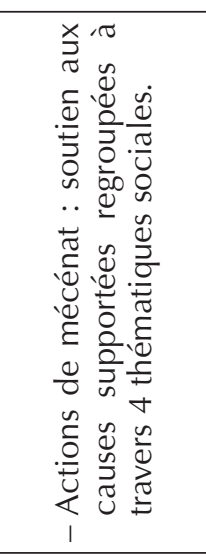 & 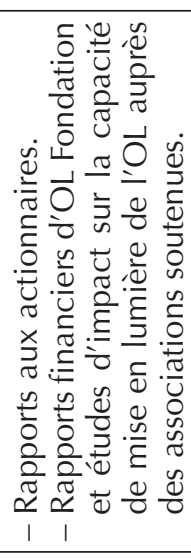 \\
\hline$\frac{\pi}{k}$ & 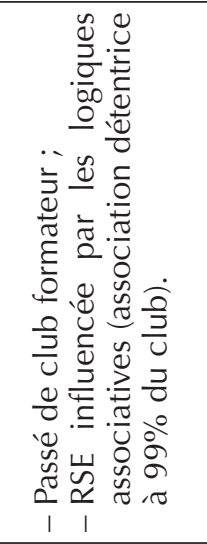 & 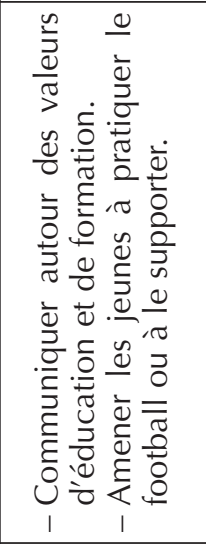 & 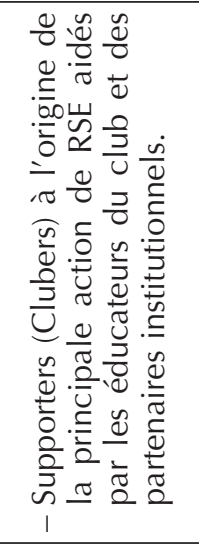 & 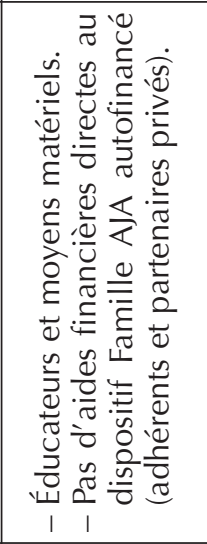 & 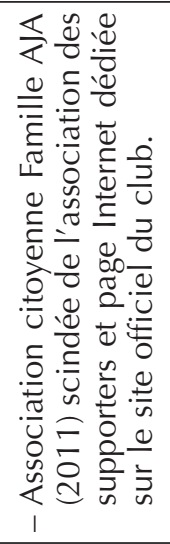 & 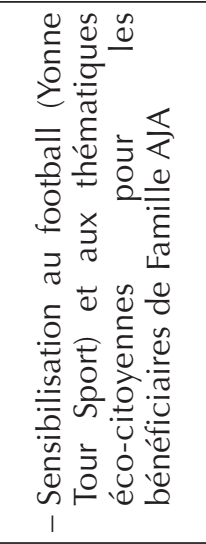 & 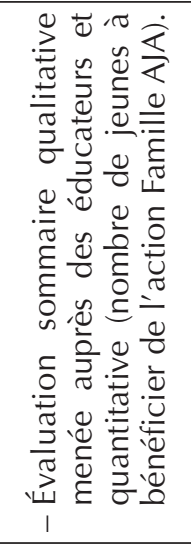 \\
\hline 岁 & 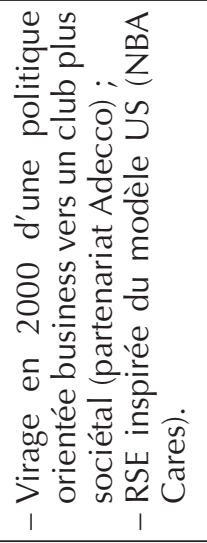 & 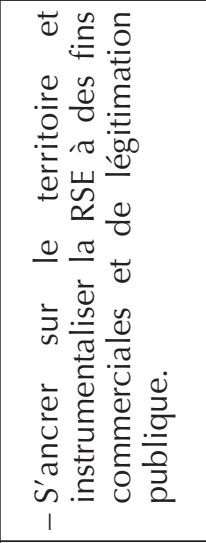 & 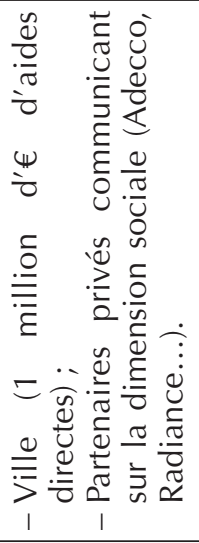 & 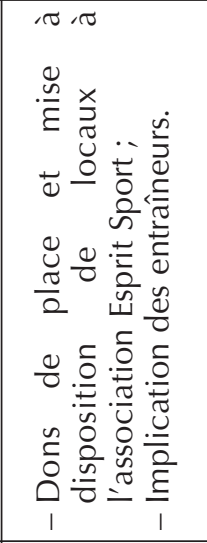 & 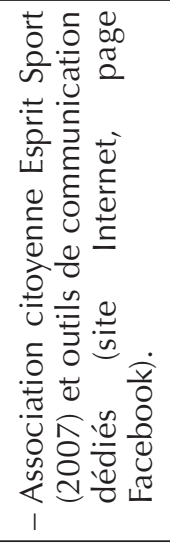 & 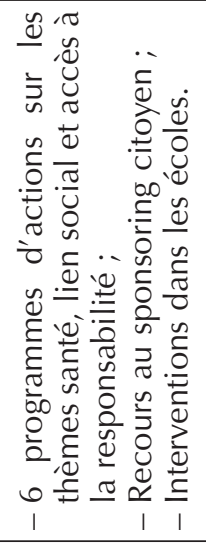 & 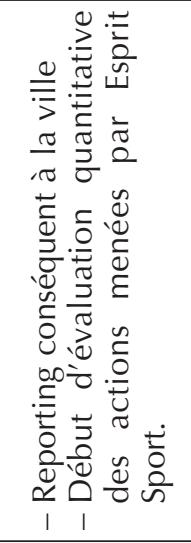 \\
\hline 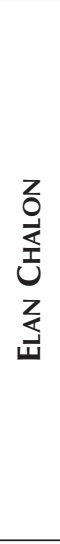 & 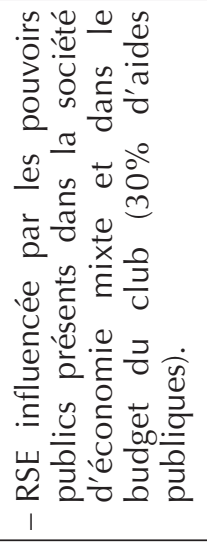 & 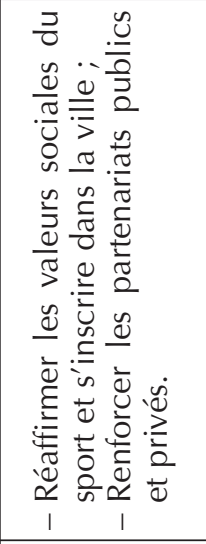 & 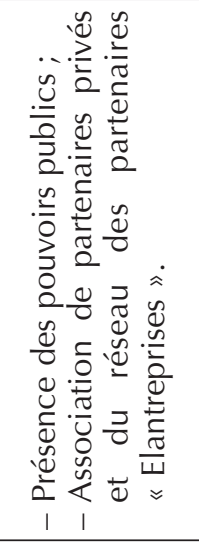 & 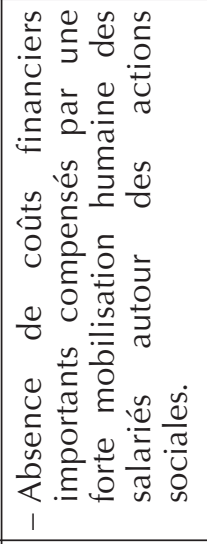 & 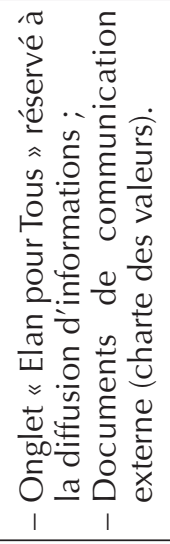 & 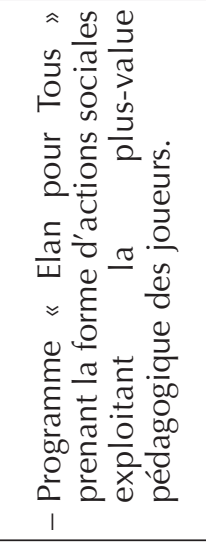 & 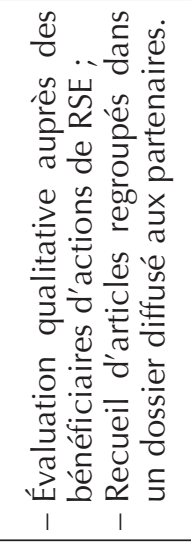 \\
\hline & 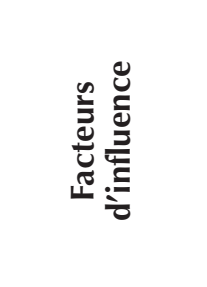 & 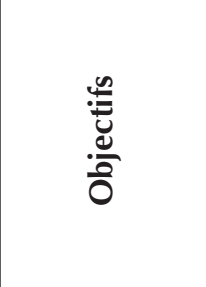 & 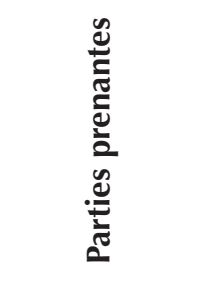 & 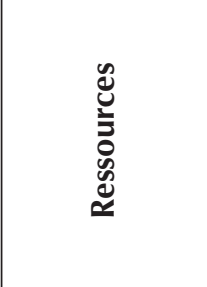 & 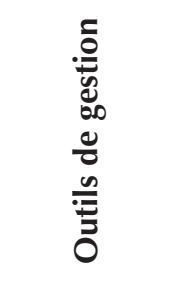 & 冚 & 岌 \\
\hline
\end{tabular}




\subsection{Facteurs d'influence : pression des partenaires publics et fort degré d'isomorphisme}

Les résultats des cas consacrent la primauté des facteurs d'influence institutionnels sur les déterminants stratégiques de l'adoption de pratiques de RSE. L'influence de la puissance publique, acteur dont l'importance a déjà été démontrée, en Amérique du nord, (Babiak \& Wolfe, 2009), et le fort degré de mimétisme des pratiques de RSE en sont les symboles.

Dans les cas de l'Elan Chalon et de l'Asvel, dont les budgets sont fortement dépendants des subventions publiques, les pratiques de RSE légitiment les subventions perçues par les collectivités dans le cadre des missions d'intérêt général (MIG) ${ }^{4}$. Piloté en partie par les élus locaux qui siègent au conseil d'administration, l'Elan Chalon perçoit un peu plus d'un millions d'euros de fonds publics $(30 \%$ du budget) au titre des MIG et participe à des actions d'éducation et d'intégration dans sa communauté. Une de leurs actions phares est une opération menée dans les quartiers sensibles de Chalon-sur-Saône dans le cadre d'un partenariat noué avec un bailleur social (Batigère). Le soutien de la ville de Villeurbanne, entrée au début des années 1990 dans le capital du club via la création d'une société d'économie mixte pour sauver l'Asvel de la relégation, est toujours palpable malgré la " privatisation » du club symbolisée par l'arrivée d'hommes d'affaires durant les années 2000. Les nombreuses actions RSE menées s'inscrivent dans la convention liant Villeurbanne et l'Asvel à laquelle le club rend régulièrement des comptes. Ses actions de proximité menées dans le domaine de la santé et du lien social sont un moyen pour les élus locaux de justifier la place de l'Asvel comme acteur social de la cité villeurbannaise, alors même qu'un lobbying intense s'exerce depuis quelques années de la part des élus de l'opposition pour délocaliser le club vers Lyon. Concernant l'OL, au-delà des aides directes et indirectes accordées par la ville, le club bénéficie d'un fort soutien politique qui prend toute son envergure dans le cadre du projet de construction du Stade des Lumières. Financé à hauteur de 450 millions d'euros par des fonds privés, ce stade, dont la déclaration d'intérêt général a été fortement soutenue par la majorité politique, a nécessité un ensemble de travaux liés à son accessibilité et estimés à environ 200 millions d'euros pris en charge par les collectivités locales. Ce soutien a poussé l'OL à s'associer, dès le début des années 2000, à un pool d'associations très impliquées dans la ville de Lyon telles que Sport dans la Ville, Docteur Clown, Handicap International) (Piché, Camy \& Chantelat, 2010). Seule l'AJA semble plutôt épargnée de la pression des partenaires publics, car le club ne touche aucune subvention dans le cadre des MIG, malgré la récente construction d'un centre de formation en 2010, dont le coût de 10 millions d'euros a été financé à hauteur de $75 \%$ par les pouvoirs publics.

Outre le poids des collectivités, l'intensité mimétique dans le secteur constitue un second déterminant important des pratiques de RSE. Le degré d'incertitude sportive qui pèse sur les clubs les a conduit à copier les meilleurs d'entre eux et a favorisé l'isomorphisme des pratiques (DiMaggio \& Powell, 1983). L'OL est allé emprunter à des modèles managériaux de grands clubs européens un certain nombre de pratiques organisationnelles. OL Fondation a ainsi été créée en 2007 , soit un an après celle de Manchester United, un des clubs dont JeanMichel Aulas, le président de l'OL, a côtoyé ses dirigeants durant son mandat à la présidence du G14 (association regroupant les 18 plus grands clubs européens dissoute en 2008 au profit de l'association européenne des clubs). L'organisation de tournois de quartiers dans le cas de l'Elan Chalon a déjà été réalisée par de nombreux clubs tels que l'Asvel, dont les premières actions de ce type remontent au début des années 2000, au moment de son partenariat citoyen tissé avec son partenaire majeur Adecco. De même, les opérations d'éducation du jeune supporter dans le cadre du programme "Famille AJA ", soutenues par le club auxerrois, ont été initiées par de nombreux autres clubs de supporters comme ceux de Lille en 2000 (Doggies) ou de Toulouse en 2005 (Média - Pitchoune) bien avant que cette initiative ne soit créée. Les modèles émergents de RSE apparaissent également fortement importés de modèles étrangers. L'Asvel a été le premier club de basket-ball français, à l'instar des franchises nordaméricaines possédant leurs propres fondations, à se doter d'une association (Esprit Sport) permettant de monter des partenariats citoyens.

\subsection{Une mise en ouvre " a minima » de la RSE guidée par des objectifs de légitimation}

Les facteurs d'influence déterminent nettement les objectifs assignés aux pratiques de RSE mises en place. Nous retrouvons un lien fort entre ces deux dimensions autour du concept de légitimité qui permet de relier les déterminants institutionnels à la

4. Ces missions relèvent d'un cadre législatif obligeant les clubs à réaliser des actions de formation, d'éducation, d'intégration ou de cohésion sociale et d'amélioration de la sécurité du public en contrepartie de subventions. 
finalité des pratiques de RSE. La réalisation de telles pratiques répond avant tout à un objectif de légitimation d'aides publiques que ce soit en matière de financement ou de choix politiques en faveur des clubs. Les pratiques de RSE demeurent assez peu intégrées aux objectifs stratégiques des clubs et constituent seulement un moyen de justification des aides perçues.

De ce point de vue, les pouvoirs publics qui exercent une influence importante sur les clubs, sont aussi la principale partie prenante à accompagner l'ensemble des clubs dans la mise en œuvre de leur démarche RSE. Les relations avec la société civile demeurent moins fréquentes, à l'exception de l'OL qui a mis sa marque à disposition d'ONG et d'associations caritatives, via sa fondation, et dans une moindre mesure de l'Elan Chalon qui a réalisé des actions de type produit-partage au profit d'associations (Playing for Change). Par ailleurs, les partenariats noués le sont souvent dans le cadre d'actions ponctuelles qui n'engagent pas les clubs à long-terme et ne font pas l'objet d'une co-construction avec les parties prenantes associées. Le rôle du club se limite à celui de " metteur en scène " d'associations parrainées et dans le meilleur des cas d'apporteur faible de ressources. Ainsi, l'AJA qui se place comme instigateur de la réalisation d'actions sociales ne fait que s'engager très faiblement au côté de "Famille AJA " en se contentant de mettre à disposition des locaux et un salarié quelques heures par semaine pour soutenir cette activité. Si les montants financiers de l'OL sont les plus importants affectés aux pratiques sociales parmi notre échantillon, l'apport d'OL Groupe demeure assez faible. Ainsi, sur la période 2007-2012, OL Groupe a financé sa fondation à hauteur d'environ 1 million d'euros (60\% du budget total de la fondation), alors que ses produits d'activités étaient estimés en cumulé à presque 1,1 milliards d'euros dans le même temps (source : rapport des documents financiers d'OL Groupe), ce qui représentait un engagement moyen d'à peine $0,1 \%$ des produits d'activité du club. Les outils de gestion employés prennent la forme d'associations (Famille AJA, Esprit Sport pour l'ASVEL), de fonds de dotation (sOLidaire) ou de fondations (OL Fondation) permettant aux clubs de communiquer aux parties prenantes externes sur leur engagement sociétal. Ces outils constituent des réceptacles aux dons permettant de financer et rationaliser les soutiens engagés auprès du secteur associatif. Souvent situés en marge du club, ils tendent principalement à augmenter la capacité d'attraction ou de fidélisation de nouveaux partenaires en couplant notamment sponsoring et mécénat afin d'assurer une communication plus responsable.
L'ensemble de ces pratiques prend forme autour d'actions de mécénat centrées principalement autour du volet sociétal et négligeant les dimensions économiques et environnementales de la RSE. Ces actions s'inscrivent la plupart du temps dans la lignée des valeurs que consacrent les discours institutionnels autour du sport que le sport est " censé " promouvoir. Les nombreux axes des programmes de RSE reprennent d'ailleurs ces thématiques. L'exemple le plus illustratif est celui de l'Asvel qui, à travers Esprit Sport, œuvre dans trois axes principaux, à savoir la santé, le lien social et l'accès à la responsabilité. Les rares actions menées dans le domaine environnemental ont été à l'initiative des clubs de l'Elan Chalon qui promeut à travers des clips promotionnels son engagement dans le développement durable, de l'AJA qui invite les jeunes supporters à respecter l'environnement et de l'OL qui avait réalisé un bilan carbone lors de la saison sportive 2009-2010, preuve d'un début de réflexion sur l'impact environnemental que peut générer un club sur son territoire. Les actions de reporting sont modestes. Dans les clubs de l'Asvel et de l'Elan Chalon, elles prennent par exemple la forme de compilations d'articles de presse destinés surtout aux pouvoirs publics commanditaires des actions de RSE. Les clubs dont la visibilité médiatique est plus importante rendent compte de leurs pratiques sur leurs sites internet, et ceux disposant d'outils de mécénat commencent à publier des rapports de leurs actions. Depuis 2008, l'OL informe du contenu des actions réalisées par sa fondation. Il publie aussi des informations sur les domaines sociaux et environnementaux comme l'article 116 de la loi NRE l'y a obligé et comme le stipule à présent le Grenelle 2 de l'Environnement. Malgré cela, l'évaluation ex ante n'apparaît pas clairement alors qu'elle pourrait, à partir de la consultation des parties prenantes, faire émerger des axes d'engagement pertinents en matière de RSE.

\section{ESSAI DE TAXONOMIE DES FORMES DE RSE}

La multiplicité des formes de pratiques de RSE nous a conduits à discuter les résultats à la lumière des typologies à l'œuvre dans le champ de la RSE. Parmi elles $^{5}$, nous avons emprunté, à celle de Martinet et Payaud (2008), des éléments afin de discuter des formes d'engagement réel et potentiel des clubs dans la voie de la RSE. Via des observations et des études de rapports de RSE de grandes sociétés cotées, ces auteurs esquissent une taxonomie des comportements

5. Nous ne pouvons dresser une liste des publications ayant consacré une typologie de la RSE car celle-ci serait trop exhaustive. Nous citons seulement là les travaux américains de Carroll (1979) et ceux français de Capron et Quairel-Lanoizelée (2010). 
stratégiques de RSE. Représentée par un continuum allant de l'entreprise " friedmanienne " (ne pratiquant pas la RSE) à l'entreprise sociale (ayant fait de la RSE sa raison d'être), la taxonomie discute de trois niveaux intermédiaires d'engagement entre ces deux idéalstypes. Le premier (RSE cosmétique) caractérise les entreprises faisant état d'une pratique légère de la RSE répondant la plupart du temps à des impératifs légaux. Le second (RSE périphérique) caractérise les entreprises faisant état de pratiques de RSE sans lien direct avec leurs stratégie. Le troisième (RSE intégrée) caractérise les entreprises faisant état d'une pratique de la RSE substantielle en lien avec leur coeur de métier. Bien que le champ d'investigation de ces chercheurs soit éloigné du nôtre, les raisons de l'adaptation de leurs travaux à notre contexte sont doubles. En plus de la proximité méthodologique avec nos travaux, leurs résultats révèlent, en effet, des stratégies de RSE dont la nature hybride s'accorde avec celle des logiques d'action des clubs sportifs professionnels.

Dans l'adaptation faite des travaux de Martinet et Payaud (2008), quatre niveaux d'engagement sont distingués (tableau 3). Nous conservons les extrémités $\mathrm{du}$ continuum (RSE friedmanienne versus sociale) envisagées comme des idéals-types. L'existence de programmes sociaux des quatre clubs étudiés laisse à penser qu'aucun comportement général ne relève de la RSE friedmanienne. A l'opposé, la forme la plus relevée d'engagement dans la RSE est proche de celle caractérisant l'entreprise " sociale " (bénéfices tournés vers la communauté). Cette dernière configuration est plus difficile à faire émerger. Premièrement, les logiques de court-terme (résultats sportifs de la semaine) prévalent dans le secteur du sport professionnel. Deuxièmement, les clubs sont détenus en majorité par des actionnaires privés intéressés par les profits indirects induits de l'activité de ces organisations (développement de réseaux d'affaires/recherche de support de communication). La discussion fait essentiellement état des possibilités de convergence des pratiques de RSE vers une forme intégrée aux logiques organisationnelles des clubs et des difficultés d'une telle mise en place.

\subsection{Vers l'émergence d'une RSE intégrée?}

À la lecture du projet de taxonomie de Martinet et Payaud (2008), l'un des traits saillants généralisable à l'ensemble des cas est le degré d'engagement cosmétique des pratiques de RSE des clubs sportifs professionnels. Celles-ci s'illustrent en effet par l'absence d'intégration qui semblent concorder avec l'idée développée par Porter et Kramer (2006) selon laquelle dans cette forme d'engagement, les pratiques de RSE sont faiblement engageante et situées "à la périphérie " des activités principales de l'organisation. Au-delà du caractère cosmétique d'une grande partie des pratiques menées, nous nous intéressons, à présent, à l'émergence de nouvelles formes plus intégrées. Nous entendons par "intégrée " toute forme de RSE permettant de diminuer les risques de découplage entre discours et pratiques. Dans notre corpus, ce niveau d'engagement caractérise les clubs ayant dépassé les simples pressions institutionnelles de l'environnement incitatrices à la mise en œuvre de «bonnes pratiques » et commencé à intégrer la RSE à leur process de management (achat, RH...). L'Asvel et l'OL, par leurs actions de RSE et leurs outils de gestion, sont des clubs pionniers dans l'engagement sociétal via leurs politiques de mécénat autour de structures dédiées. Le club lyonnais a ainsi élaboré un plan stratégique autour d'axes symbolisant les valeurs fortes du club dans lesquels la RSE y est mentionnée comme "une composante intégrée à tous les niveaux $d u$ club (qui) se formalise notamment par la mise en place d'actions de solidarité ou encore par le soutien à des projets innovants" (rapport OL Groupe 2012).

L'un des exemples de RSE intégrée, à même de coupler stratégie de RSE et logiques économiques des clubs, concerne le domaine de la formation. Rarement mentionnée comme objectif lié à la réalisation de pratiques sociétales, la formation au sens large (staff administratif et technique) apparaît pourtant comme l'exercice d'une RSE "implicite " (Matten et Moon, 2008). Elle constitue en effet une opportunité stratégique pour les clubs de déconstruire la dichotomie entre l'économique et le social. Les clubs étudiés se sont historiquement ou récemment investis dans ce domaine. À titre d'exemple, l'OL a même été mandaté par le Ministère de l'Economie pour expertiser la protection et la valorisation du savoir-faire de la formation dans le football afin d'éditer un label distinguant " les centres de formation ayant certaines pratiques cohérentes avec l'idée de responsabilité sociétale $»^{6}$.

\subsection{Les difficultés d'une RSE intégrée}

L'alignement entre une RSE " déclarée » et "pratiquée " demeure difficile à obtenir. De là naissent des situations dites de "découplage " (Crilly, Zollo \& Hansen, 2012) faisant peser un flou sur la capacité des organisations à intégrer pleinement la RSE. Il en est de même pour les clubs sportifs professionnels qui, paradoxalement, ont des 


\begin{tabular}{|c|c|c|c|c|c|c|c|}
\hline 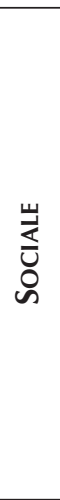 & 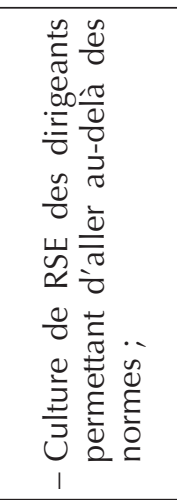 & 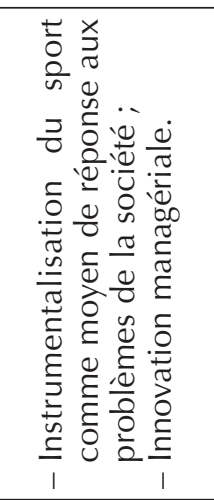 & 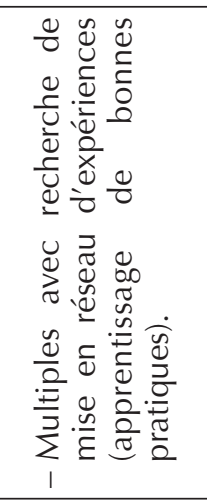 & 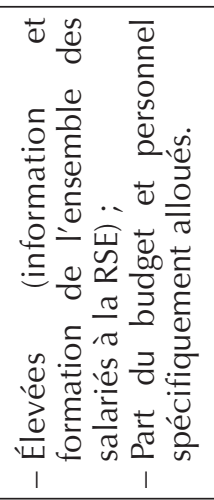 & 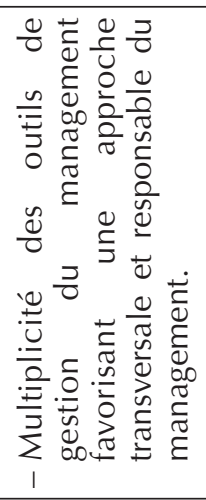 & 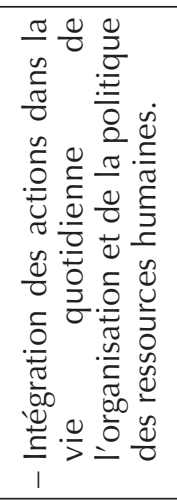 & 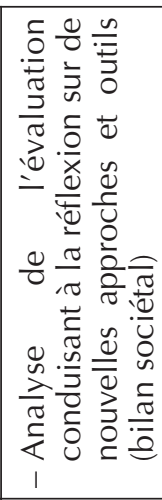 \\
\hline $\begin{array}{l}\text { 㟥 } \\
\text { 岀 } \\
\underline{\underline{z}}\end{array}$ & 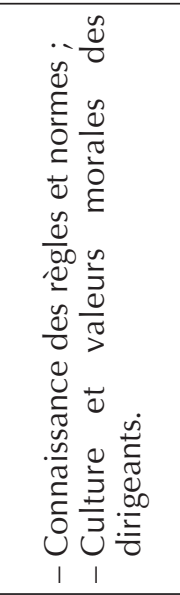 & 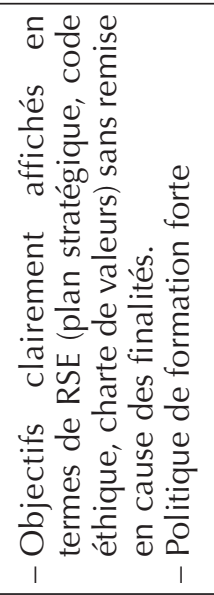 & 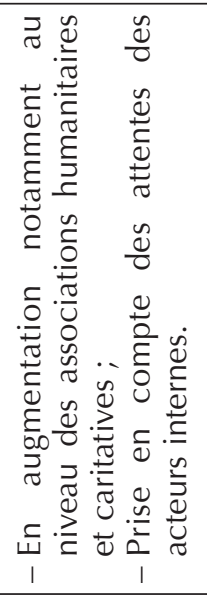 & 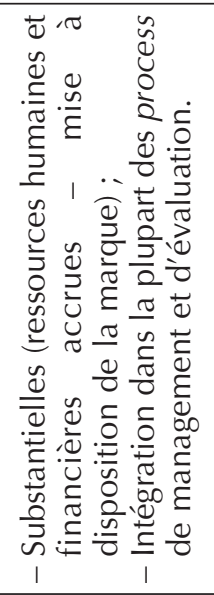 & 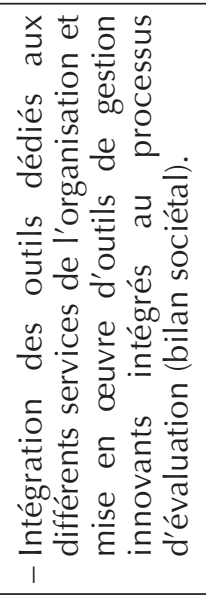 & 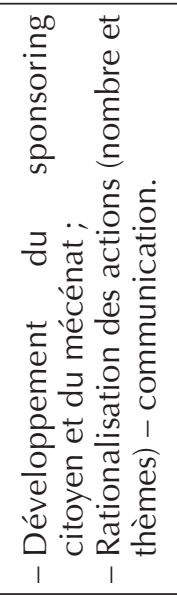 & 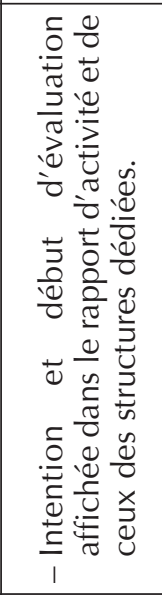 \\
\hline 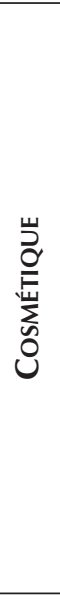 & 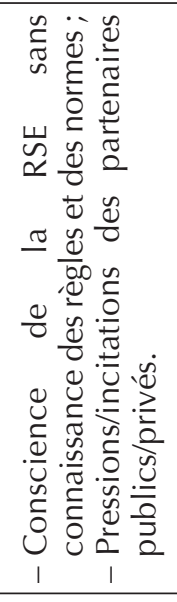 & 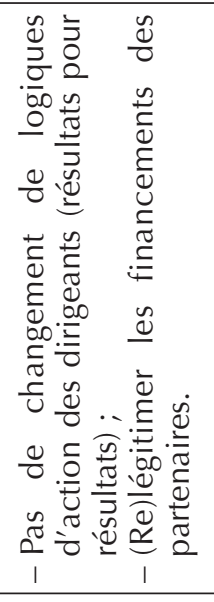 & 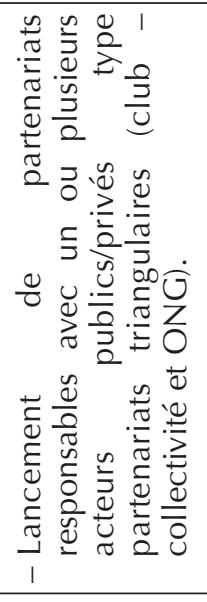 & 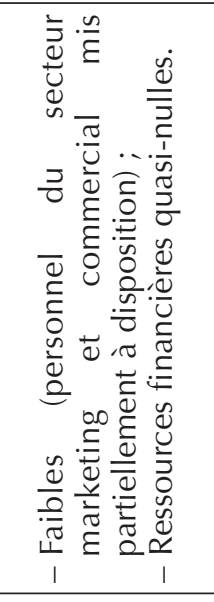 & 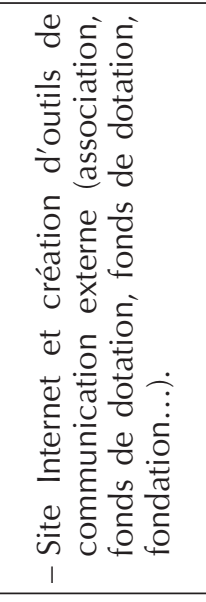 & 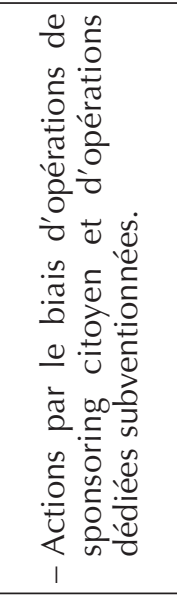 & 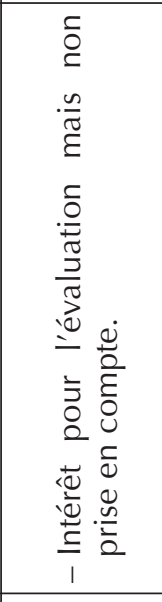 \\
\hline 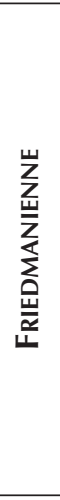 & 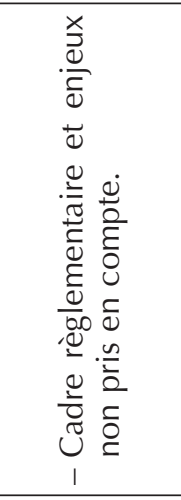 & 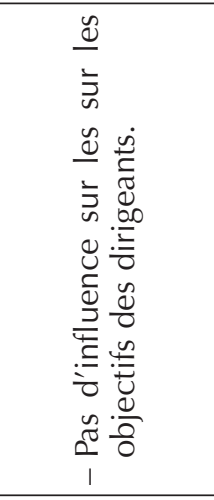 & 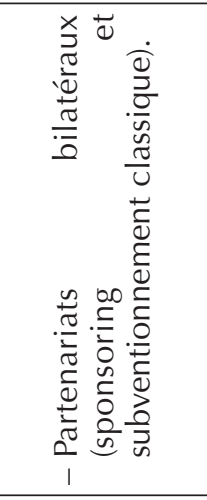 & 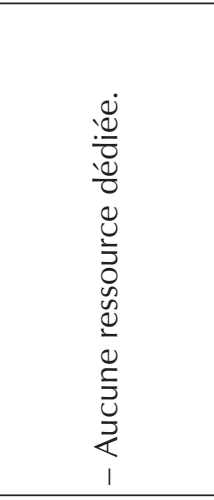 & 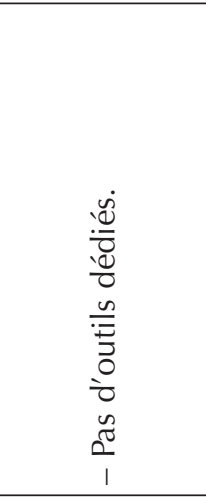 & 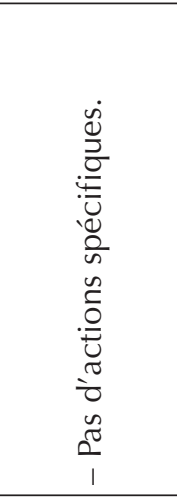 & 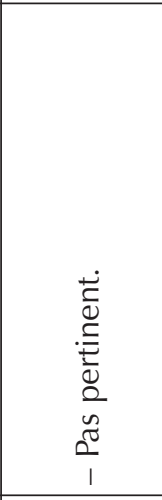 \\
\hline & 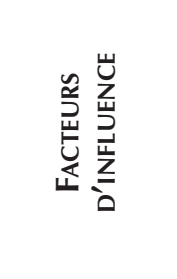 & 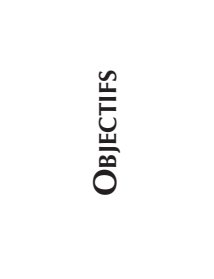 & 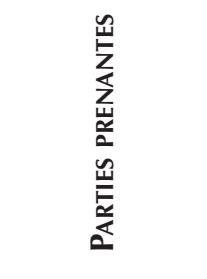 & 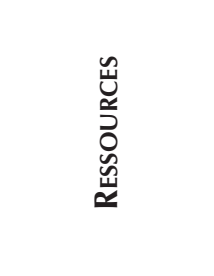 & 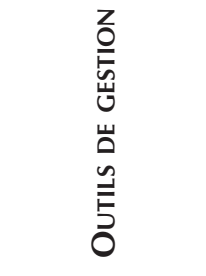 & $\underline{E}$ & 旁 \\
\hline
\end{tabular}


difficultés à intégrer cette composante à leur stratégie globale. A titre d'exemple, l'Elan Chalon et l'AJA, dont les logiques organisationnelles (statuts juridiques, modes de gouvernance...) sont proches de celles des entreprises du champ de l'économie sociale et solidaire (ESS), n'ont pas encore exploité toute la création de valeur potentielle que peut engendrer une intégration de la RSE à leur développement. L'AJA a dû faire face, dans les années 2010, à des querelles internes marquées par des luttes et changements de gouvernances ainsi que des difficultés sportives qui ont retardé la prise de conscience des enjeux de la RSE, faisant de ce club le moins engagé de tous dans cette voie. En revanche, si l'Elan Chalon montre des prémisses d'intégration de la RSE, les raisons de la difficulté de s'engager pleinement dans cette voie apparaissent différentes. Ce club se heurte à des déficits de ressources tant matérielles qu'humaines pour le faire et laisse planer des doutes sur la capacité d'organisations de plus petites tailles à le faire.

Des clubs comme l'Asvel ou l'OL, dont les finalités s'orientent plus vers la recherche de profits (multiples actionnaires, présence d'holding...) sont aussi confrontés à des problématiques de découplage fortes. A l'OL, les échecs répétés du projet précédent orienté " business " (construction du stade retardée, effondrement de l'action de 24 à 2 euros, mauvais choix d'achats/ventes de joueurs fin des années 2000, absence de décollage économique de la marque OL...) ont poussé les dirigeants à repenser le modèle de développement. Il en est de même pour l'Asvel qui a opéré un virage brusque au début des années 2000 après une période marquée par un désencastrement fort du club dans sa localité (prise de participation d'actionnaires privés, recrutement de joueurs mercenaires européens...). Ces contextes tendent à instrumentaliser les pratiques de RSE et conduisent à s'interroger sur les véritables desseins qui sont à l'origine de ses pratiques.

\section{CONCLUSION}

L'étude des pratiques de RSE dans le domaine du sport professionnel français fait apparaître des perspectives de recherches dans le champ des organisations " hybrides » dont les développements en matière de RSE sont féconds ces dernières années (Battilana \& Dorado, 2010 ; Crilly, Zollo \& Hansen, 2012). La lecture faite des pratiques de RSE à partir du cadre d'analyse est transposable aux organisations dont les logiques d'actions hybrides sont comprises entre l'économique et le social. Un des apports de cet article tient à l'originalité et la globalité du cadre d'analyse. En effet, ce dernier combine approches néo-institutionnelle (Campbell, 2007) et stratégique (Porter \& Kramer, 2006) dont l'association révèle les tensions à l'œuvre dans ces organisations. Cet article fait ainsi écho aux travaux en matière de responsabilité sociale des organisations (RSO), concept jugé moins réducteur que celui de RSE (Bento, 2009). En ce sens, le champ d'investigation constitué par les clubs professionnels apparaît comme nouveau terrain d'étude de la RSO en sciences de gestion.

L'analyse des pratiques de RSE des clubs sportifs professionnels français montre que leur forme est essentiellement cosmétique. L'adoption de ces pratiques est, avant tout, le fruit de pressions institutionnelles, notamment de la part des pouvoirs publics par le biais d'aides financières directes ou indirectes (soutien politique) ainsi que d'une forte intensité mimétique inhérente au secteur. La légitimité apparaît l'objectif central de la réalisation des pratiques de RSE expliquant le versant exclusivement externe des formes de RSE développées, comme le montre la multiplication des actions de sponsoring citoyen luttant contre l'image du sport business et de ses dérives. Si la recherche de légitimité apparaît être une quête perpétuelle des dirigeants de clubs tant leur devoir de (re)légitimer leur rôle historiques d'acteurs " responsables » dans et pour la cité devient incontournable (Durand \& Bayle, 2004), celle-ci peut pourtant participer d'une vision plus proactive. A partir de l'adaptation de la typologie esquissée par Martinet et Payaud (2008), les possibilités de convergence vers une RSE intégrée apparait stratégique. Celle-ci peut en effet participer à regrouper un ensemble de parties prenantes des clubs (sponsors, collectivités publiques, salariés, supporters...) autour d'objectifs sociétaux et non plus uniquement sportifs.

Paradoxalement, les clubs sportifs professionnels dont les logiques d'action sont finalement assez proches de celles des organisations du champ de l'ESS ont des difficultés à intégrer la RSE. Alors que le pouvoir médiatique du sport ouvre des univers de possibilités, de créativité et d'impacts multiples très riches pour les parties prenantes et les territoires d'un club professionnel, les dérives du secteur (argent roi, dopage, corruption...) remettent perpétuellement en cause cette finalité. Le faible degré d'engagement des clubs dans la voie de la RSE est toutefois à relativiser, notamment eu égard aux impacts négatifs engendrés par certaines grandes multinationales qui, en dépit de ressources suffisantes pour réaliser des pratiques ambitieuses en matière de RSE ne le font pas toujours. Cette contribution interroge plus globalement la responsabilité du management de ces organisations et la nature de la performance qui les guide. L'engagement des clubs dans la voie de la RSE se veut une alternative aux modèles économiques des clubs sportifs professionnels évalués à la lueur de leurs seules performances sportives et financières. L'intérêt de mesurer la performance sociétale et de rendre des comptes apparaît ainsi nécessaire. De même, l'article invite à penser la RSE comme un nouvel élément de 
régulation du sport professionnel. Dans cette voie, les différentes organisations $\mathrm{du}$ secteur sportif (fédérations nationales, internationales et ligues professionnelles) pourront considérer à l'avenir le pouvoir normatif de la RSE afin de s'assurer de véritables contenus adossés à la mise en place de pratiques dites « responsables».

\section{BIBLIOGRAPHIE}

Acquier, A. 2007. RSE et outils de gestion : perspectives pour l'analyse des marchés de l'évaluation extra-financière. Revue de l'Organisation Responsable, 2 (2): 5-15.

Acquier, A., \& Gond, J-P. 2007. Aux sources de la responsabilité sociale de l'entreprise : (re)lecture et analyse d'un ouvrage fondateur : Social responsibilities of the businessman d'Howard Bowen, 1953. Finance-Contrôle-Stratégie, 10(2): 5-35.

Aguinis, H. \& Glavas, A. 2012. What we know and don't know about CSR: a review and research agenda. Journal of Management, 38 (4): 932-968.

Babiak, K., \& Trendafilova, S. 2011. CSR and environmental responsibility: motives and pressures to adopt green management practices. Corporate Social Responsibility and Environmental Management, 18: 11-24.

Babiak, K., \& Wolfe, R. 2009. Determinants of corporate social responsibility in professional sport: internal and external factors. Journal of Sport Management, 23 (6): 717-742.

Bardin, L. (2001). L'analyse de contenu. Paris: Presses Universitaires de France.

Battilana, J., \& Dorado, S. 2010. Building sustainable hybrid organizations: the case of commercial microfinance organizations. Academy of Management Journal, 53 (6): 1419-1440.

Bayle, E. 2007. Essai de définition du management des organisations sportives : objet, champ, niveaux d'analyse et spécificités des pratiques managériales. Revue Staps, 75 (1): 59-81.

Bayle, E., Chappelet, J-L., François, A. \& Maltèse, L. 2011. Sport et RSE. Vers un management responsable ? Bruxelles: De Boeck Editions.

Bayle, E., \& Mercier, S. 2008. Sport et éthique : enjeux et outils pour le marketing sportif. Revue Française du Marketing, 219 (4/5): 9-26.

Bento, L. 2009. De la CSR-corporate social responsibility à la RSO-responsabilité sociale des organisations. Quelques apports à la réflexion. Management \& Avenir, 3 (23): 73-82.

Boiral, O. 2006. La certification ISO 14001 : une perspective néo-institutionnelle. Management International, 10 (3): 67-79.

Borchani-Maktouf, M. 2010. Déterminants de la stratégie sociétale des multinationales en France. Revue de l'organisation responsable, 5 (2): 39-58.
Bowen, H. R. 1953. Social Responsibilities of the Businessman. New York: Harper \& Row.

Bradish, C.L. \& Cronin, J. J. 2009. Corporate social responsibility in sport. Journal of Sport Management, 23 (6): 691-697.

Branco, M. C., \& Rodrigues, L. L. 2006. Corporate social responsibility and resource-based perspectives. Journal of Business Ethics, 69 (2): 111-132.

Breitbarth, T., \& Harris, P. 2008. The role of corporate social responsibility in the football business: towards the development of a conceptual model. European Sport Management Quarterly, 8 (2): 179206.

Campbell, J. L. 2007. Why would corporations behave in socially responsible ways? An institutional theory of corporate social responsibility. Academy of Management Review, 32 (3): 946-967.

Capron, M., \& Quairel-Lanoizelée, F. 2007. La responsabilité sociale d'entreprise. Paris: La Découverte.

Carroll, A. B. 1979. A Three-Dimensional Conceptual Model of Corporate Performance. Academy of Management Review, 4 (4): 497-505.

Carroll, A.B. 1999. Corporate Social Responsibility: Evolution of a Definitional Construct. Business \& Society, 38 (3): 268-295.

Child, J. 1997. Strategic choice in the analysis of action, structure, organizations and environment: retrospect and prospect. Organization Studies, 18 (1): 43-76.

Crilly, D., Zollo, M., \& Hansen, M. 2012. Faking it or muddling through? Understanding decoupling in response to stakeholder pressures. Academy of Management Journal, 55 (6), 1429-1448.

Desreumaux, A. 2005. Théorie des organisations. Paris: Editions EMS.

DiMaggio, P. J., \& Powell, W. W. 1983. The iron cage revisited: institutional isomorphism and collective rationality in organizational fields. American Sociological Review, 48 (2): 147-160.

Dupuis, J-C. 2011. Le management responsable. Un modèle de gestion de l'obsolescence morale. Revue Française de Gestion, 215 (6): 69-85.

Durand, C., \& Bayle, E. 2004. Soutien de la sphère publique locale aux clubs sportifs professionnels : le retour du club citoyen ? In S. Cueille, R. Le Duff \& $\mathrm{J}-\mathrm{J}$. Rigal (Eds.), Management local, de la gestion à la gouvernance: 211-231. Paris: Dalloz.

Eisenhardt, K. M. 1989. Building theories from case study research. Academy of Management Review, 14 (4): $532-550$

Freeman, R. E. 1984. Strategic management: A stakeholder approach. Boston: Pitman.

Glaser, B. G., \& Strauss, A. L. 1967. The discovery of grounded theory. Hawthorne: Aldine Press.

Godfrey, P. C. 2009. Corporate social responsibility in sport: an overview and key issues. Journal of Sport Management, 23 (6): 698-716. 
Gond, J-P., \& Igalens, J. 2012. Manager la responsabilité sociale de l'entreprise. Paris: Pearson. Hasselbladh, H., \& Kallinikos, J. 2000. The project of rationalization: a critique and reappraisal of neoinstitutionalism in organization studies. Organization studies, 21 (4): 697-720.

Husted, B. W., \& Allen, D. B. 2000. Is it ethical to use ethics as strategy? Journal of Business Ethics, 27 (1/2): 21-31.

Judge, W. Q., \& Zeithaml, C. P. 1992. Institutional and strategic choice perspectives on board involvement in the strategic decision process. Academy of Management Journal, 35 (4): 766-794.

Kuper, S. \& Szymanski, S. 2012. Les attaquants les plus chers ne sont pas ceux qui marquent le plus (ouvrage traduit). Bruxelles: De Boeck Editions.

Le Borgne-Larivière, M., Mauléon, F., \& Schier, G. 2009. Une lecture néo-institutionnaliste des pratiques de RSE : le cas des chartes éthiques. Management \& Avenir, 23 (3): 187-198.

Lee, M-D. P. 2008. A review of the theories of corporate social responsibility: Its evolutionary path and the road ahead. International Journal of Management Reviews, 10 (1): 53-73.

Maltese, L. 2009. Managing assets in the field of sports special events: proposal for a new methodological and analytical approach. International Journal of Sport Management and Marketing, 5 (3): 310-329.

Maon, F., Lindgreen, A., \& Swaen, V. 2009. Designing and implementing corporate social responsibility: an integrative framework grounded in theory and practice. Journal of Business Ethics, 87: 71-89.

Martinet, A-C., \& Payaud, M. A. 2008. Formes de RSE et entreprises sociales. Une hybridation des stratégies. Revue Française de Gestion, 180 (1): 199214.

Matten, D., \& Moon, J. 2008. "Implicit" and "explicit" CSR: A conceptual framework for a comparative understanding of corporate social responsibility. Academy of Management Review, 33 (2): 404-424.

McWilliams, A., Siegel, D. S., \& Wright, P. M. 2006. Corporate social responsibility: strategic implications. Journal of Management Studies, 43 (1): 1-18.

McWilliams, A., \& Siegel, D. S. 2011. Creating and capturing value: strategic corporate responsibility, resource-based theory, and sustainable advantage. Journal of management, 37 (5): 1480-1495.

Meyer, J. W., \& Rowan, B. 1977. Institutionalized organizations: formal structure as myth and ceremony. The American Journal of Sociology, 83 (2): 340-363.

Miles, M. B., \& Huberman, A. M. 2003. Analyse des données qualitatives. Bruxelles : De Boeck.

Oliver, C. 1991. Strategic responses to institutional processes. Academy of Management Review, 16 (1): 145-179.
Paradas, A. 2008. La position des petites entreprises face à la responsabilité sociale. Revue de l'Organisation Responsable, 3 (1): 39-52.

Paramio-Salcines, J.L., Babiak, K., \& Walters, G. 2013. The Routledge handbook of sport and corporate social responsibility. New-York: Routledge.

Pastore-Chaverot, M. 2011. Les stratégies RSE des grandes entreprises : les facteurs d'influence. Analyse des discours des entreprises du CAC 40. Thèse non publiée de doctorat de gestion, Université Paul Verlaine, Metz.

Piché, S., Camy, J., \& Chantelat, P. 2010. Les transferts économiques des clubs sportifs professionnels vers les organisations caritatives : comparaison des situations au Canada et en France. Revue Staps, 2 (88): 99-113.

Pfeffer, J., \& Salancik, G. R. 1978. The external control of organizations: a resource dependence perspective. New York: Harper \& Row.

Porter, M. E., \& Kramer, M. R. 2006. Strategy and society: the link between competitive advantage and corporate social responsibility. Harvard Business Review, 84 (12): 78-92.

Porter, M. E., \& Kramer, M. R. 2011. Creating shared value. Harvard Business Review, 89 (1/2): 62-77.

Quairel-Lanoizelée, F. 2011. ISO 26000 : une convention de qualité ou chronique d'une norme "dans les normes". In M. Capron, F. QuairelLanoizelée \& M-F. Turcotte (Eds.), ISO 26000 : une norme "hors norme" ?: 113-130. Paris: Economica.

Scott, W. R. 1995. Institutions and organizations. Thousand Oaks: Sage Publications.

Sheth, H., \& Babiak, K. 2010. Beyond the game: perceptions and practices of corporate social responsibility in the professional sport industry. Journal of Business Ethics, 91 (3): 433-450.

Smith, A. C. T., \& Westerbeek, H. M. 2007. Sport as a Vehicle for Deploying Corporate Social Responsibility. Journal of Corporate Citizenship, 25: 43-54.

Suchman, M. C. 1995. Managing legitimacy: strategic and institutional approaches. Academy of Management Review, 20 (3): 571-610.

Vogel, D. 2005. The market for virtue. The potential and limits of corporate social responsibility. Washington DC: Brookings Institution Press.

Walker, M., Kent, A., \& Vincent, J. 2010. Communicating socially responsible initiatives: an analysis of US professional teams. Sport Marketing Quarterly, 19 (4): 187-195.

Walters, G., \& Chadwick, S. 2009. Corporate citizenship in football: delivering strategic benefits through stakeholder engagement. Management Decision, 47 (1): 51-66.

Wood, D.J. 1991. Corporate social performance revisited. Academy of Management Review, 16 (4): 691-718.

Yin, R.K. 2003. Case study research: design and methods. Thousand Oaks: Sage Publications. 


\section{ANNEXE 1 : GUIDE DES ENTRETIENS7.}

Le guide d'entretien se composait d'une phrase d'entame du type "Nous aimerions savoir ce que représente la responsabilité sociale d'un club comme le vôtre ?" suivie d'une étape de questionnement autour des sept dimensions du cadre d'analyse dont les principales questions pour chacune d'entre elles, sont présentées cidessous.

\begin{tabular}{|c|l|}
\hline ThèMES ABORDÉs & \multicolumn{1}{c|}{ QUESTIONS CENTRALES } \\
\hline Facteurs d'influence & $\begin{array}{l}\text { - Quels ont été les déterminants qui vous ont poussé à entreprendre des pratiques de type } \\
\text { RSE ? }\end{array}$ \\
\hline Objectifs & - Quels objectifs étaient poursuivis quant à la réalisation de ce type d'actions ? \\
\hline Parties prenantes & $\begin{array}{l}\text { - Est-ce qu'il y a des parties prenantes qui vous ont poussé à vous engager sur la voie de } \\
\text { la RSE ? }\end{array}$ \\
\hline Ressources & - Quelles ressources avez-vous engagées dans la réalisation de ces pratiques ? \\
\hline Outils de gestion & $\begin{array}{l}\text { - Quels outils de gestion avez-vous mobilisé dans la mise en place et le suivi des } \\
\text { pratiques de RSE ? }\end{array}$ \\
\hline Actions & - Quelles ont été les principales actions réalisées? \\
\hline Évaluation & - Disposez-vous de moyens permettant d'évaluer vos pratiques de RSE ? \\
\hline
\end{tabular}

7. Nous ne faisons ici que mentionner les grandes étapes de l'entretien. Seules les questions centrales sont indiquées et masquent de nombreuses questions de relance utilisées dans l'approfondissement de chacune des dimensions. 